\title{
A EXPANSÃO ARGUMENTATIVA NA ESCRITA DE MULTIMODAL ESSAYS NA GRADUAÇÃO EM LETRAS LÍNGUA INGLESA
}

\author{
LEANDRA DIAS 1 \\ MARIA CRISTINA DAMIANOVIC ${ }^{2}$
}

Programa de Pós-Graduação em Letras, Universidade Federal de Pernambuco

lleandradias2013@gmail.com ; mcdamianovic@gmail.com

\begin{abstract}
Resumo. Este artigo objetiva discutir a relevância da multimodalidade (KRESS, 2003) na expansão da argumentação (LIBERALI, 2013) quando da produção escrita de Multimodal Essays na graduação de Letras - Língua Inglesa (Lic.), o que implica investigar a relevância e a contribuição que a Pedagogia dos Multiletramentos (KALANTZIS; COPE, 2009), com foco na multimodalidade de textos, pode trazer para o ensino de língua estrangeira no contexto universitário. Essa discussão emerge de uma inquietação relacionada à distância entre as práticas de linguagem em contextos acadêmicos e o uso real da língua na Modernidade Recente (MOITA LOPES, 2013). Surge de uma proposta de trabalho realizada na UFPE, na graduação em Letras-Inglês (Lic.) pelo grupo de pesquisa LIGUE/CNPq (Linguagem, Língua, Escola e Ensino), com o intuito de aproximar as práticas pedagógicas de ensino de língua inglesa das necessidades de linguagem dos alunos nos tempos atuais. Os dados focais deste estudo estão voltados para a experiência vivenciada na disciplina de Inglês VI, no curso de Letras-Inglês (Lic.), baseada na Pedagogia dos Multiletramentos com foco na multimodalidade. O corpus discutido é composto por três versões de um multimodal essay escrito por um aluno focal. Na discussão da análise, nos voltamos para a expansão argumentativa ocorrida na escrita em três versões desses textos focais, tendo como elementos norteadores alguns dos aspectos enunciativos, discursivos e linguisticos da linguagem argumentativa (LIBERALI, 2013). As análises iniciais demonstram que o entrelaçamento dos estudos sobre Argumentação e Multimodalidade pode se configurar como uma resposta interessante para as recentes demandas teóricometodológicas emergentes da sala de aula atual.
\end{abstract}

Palavras-chave: produção escrita; multimodalidade; expansão argumentativa; ensino; língua inglesa.

\footnotetext{
${ }^{1}$ Leandra Dias é editora chefe da Revista Pearson English, bem como coordenadora do projeto de formação profissional de professores de língua inglesa da Pearson Brasil (Webinars Pearson). Mestre em Linguística (PPGL - UFPE) e pesquisadora do Grupo de Pesquisa LIGUE (Linguagem, Línguas, Escolas e Ensino), atualmente trabalha como consultora acadêmica da Pearson Brasil e coordenadora do grupo de estudos MEL: Multiletramentos e Estudos de Linguagem.

${ }^{2}$ Maria Cristina Damianovic é professora do Departamento de Letras e do Programa de Pós-Graduação em Letras da UFPE. Coordena o Grupo de Pesquisa LIGUE: Linguagem, Línguas, Escola e Ensino. Possui Mestrado e Doutorado pelo LAEL/PUC-SP. Realiza estágio de Pós-Doutorado no LAEL/PUC-SP com a supervisora Profa. Dra. Fernanda Liberali, coordenadora do Grupo de Pesquisa LACE: Linguagem em Contextos Escolares, e professora no LAEL/PUC-SP. http://ufpe.academia.edu/mariacristinadamianovic
} 


\begin{abstract}
This article aims to discuss the relevance of Multimodality (KRESS, 2010) in the expansion of argumentation (LIBERALI, 2013) in written multimodal essays in the Language Arts Undergraduate course. This implies investigating the relevance and contribution the Pedagogy of Multiliteracies (KALANTZIS; COPE, 2009), focusing on multimodality of texts, can bring to the teaching of foreign languages in an academic context. This discussion emerges from a concern about the gap between language practice in academic contexts and the real use of language in Recent Modernity (MOITA LOPES, 2013). It is based on a project which took place at UFPE, on the Language Arts Undergraduate course conducted by the research group LIGUE/CNPq (languages, school and teaching) focused on uniting pedagogical practices and the real needs of current times students. The corpus discussed is composed of three versions of a multimodal essay written by one of the focal students. For the data analysis, we focus on the argumentative expansion that occurred throughout the three versions of this focal multimodal essay, having as guiding elements the enunciative, discursive and linguistics aspects of the argumentative language (LIBERALI, 2013). Initial analysis showed that the intertwining of Argumentation and Multimodality studies can be conceived as an interesting response to the recent emerging theoretical-methodological demands of the current classroom.
\end{abstract}

Keywords: writing; multimodality; argumentative expansion; teaching; language.

\title{
INTRODUÇÃO
}

Temos que voltar os nossos pensamentos para o mundo que estamos vendo, vivendo.

(Rajagopalan, 2011)

Em suas colocações sobre a consolidação da Linguística Aplicada no Brasil, Moita Lopes (2013) faz considerações pontuais sobre as transformações sociais provocadas pelo processo de globalização no período histórico que denomina Modernidade Recente. Segundo o autor, essa denominação é utilizada para descrever o período da história contemporânea relacionado às últimas décadas do século $\mathrm{XX}$ e os tempos em que vivemos, quando é notório o turbilhão de mudanças de ordem econômica, política, tecnológica e, consequentemente social. Sentimentos de ordem vertiginosa parecem ser característicos desse momento, que faz emergir questionamentos sobre crenças, conhecimentos e o posicionamento social do homem na sociedade em que vive.

Mudanças tão intensas orientam novos paradigmas de pensamento e, consequentemente, a produção de linguagem dos sujeitos que vivem essas transformações. Trazendo nossas preocupações para a educação na graduação em Licenciatura em Letras - Língua Inglesa (Lic.), concordamos com Moita Lopes (2013) quando este aponta para a urgência do surgimento de uma consciência crítica que aborde as necessidades de inovações teórico-metodológicas que compreendam a sala de aula, os 
professores, os alunos, os materiais e métodos de ensino em uma perspectiva sóciohistórico-cultural condizente com a complexidade do momento atual. No que diz respeito à linguagem, esta precisa ser utilizada como um artefato cultural capaz de orientar metodologias de ensino-aprendizagem democráticas, que reflitam as necessidades de participação real nas atividades sociais do indivíduo desta época da história humana.

Segundo o autor supracitado, vivemos a era da hipersemiotização do uso da linguagem, do homem moderno em constante movimento em sociedades hipersemiotizadas. Ou ainda, segundo Rojo (2013), a era das linguagens líquidas, na qual o homem experimenta as mais diversas possibilidades de escolhas para criação de significados. Estas oportunizadas pelas relações comunicativas provenientes do mundo digital e suas múltiplas semioses (hipertexto, áudios, imagens, vídeos etc.).

Dessa forma, enquanto há quase duas décadas "a competência em relação a um único modo, a escrita, era vista como suficiente para a tarefa de compor um texto, precisamos entender agora, os potenciais semióticos de todos os modos que envolvem a criação do texto multimodal" (BEZEMER; KRESS, 2010, p. 11). Isso significa que é salutar colocar o texto verbal em relação com um conjunto de signos de outras mo $^{3}$ dalidades (imagem estática, imagem em movimento, som, fala) que o cercam, ou intercalam ou impregnam, assim como afirma Rojo (2013).

Em virtude da orientação hipersemiotizada do uso da linguagem decorrente da crescente conexão global e da facilidade de acesso às mais exóticas diversidades culturais, a tarefa de compor enunciados e interagir com eles e com o mundo mudou. A queda dos muros que restringiam o intercâmbio cultural e linguístico entre povos oportuniza a constante (re)criação de significados sobre o mundo através de perspectivas plurilinguísticas, multissemióticas e pluriculturais (ROJO, 2012). Essas perspectivas urgem que a escola também seja um espaço para a compreensão e produção plurilinguística baseada na multissemiose pluralista das culturas.

Nesse panorama, voltamos nossas inquietações para o ambiente de ensino de Língua Inglesa na graduação em Letras (Lic.) da UFPE, que se realizava por meio de um ensino da produção escrita discente pautada tradicionalmente na materialidade linguística. Nos últimos dois anos, na tentativa de aproximar as práticas de linguagem acadêmicas à vida real dos alunos, há, desde então, uma busca por produções escritas plurilinguísticas, multissemióticas e pluriculturais, que reflitam os usos de linguagem real da sociedade na qual estamos inseridos. Isso é o resultado de um esforço docente que possibilite aos alunos acesso a uma educação linguística que lhes permita se integrarem plenamente à vida pública, comunitária e acadêmica do meio social de que fazem parte.

Por isso que concordamos com Kalantzis e Cope (1996/2000) quando afirmam que a ênfase sobre inovação e criatividade pode se encaixar bem com uma pedagogia que vê a linguagem e outros modos de representação como dinâmicos, refeitos constantemente por criadores de significado na mudança e em variados contextos. A escola - no caso deste estudo, a universidade, o curso de Letras-Língua Inglesa (Lic.) e mais especificamente, as disciplinas voltadas para a produção escrita - precisariam

\footnotetext{
3 "Where up to two decades ago maybe, competence in relation to one mode, writing, was seen as sufficient for the task of composition of text, we now need to understand the semiotic potentials of all modes involved in the design and making of multimodal text."
} 
abordar a língua em seus diversos feixes de realizações, levando em consideração os aspectos multimodais e multiculturais (ROJO, 2012) dos letramentos na atualidade.

\section{A PEDAGOGIA DOS MULTILETRAMENTOS NA LICENCIATURA EM LÍNGUA INGLESA NA CONTEMPORANEIDADE}

O mundo estava mudando, os ambientes de comunicação estavam mudando e isso significava para nós que, para seguir essas mudanças, o ensino e a aprendizagem de letramento precisariam mudar também.

(Kalantzis; Cope, 2009)

A necessidade de uma pedagogia que tratasse a linguagem por meio de uma perspectiva plural, global e multissemiótica foi objeto de debate de um grupo de pesquisadores, o Grupo de Nova Londres, doravante GNL, que, em 1996, se reuniu para discutir, entre outras coisas, como a linguagem entra em harmonia com a diversidade linguística e cultural.

O Grupo de Nova Londres foi incialmente composto por dez educadores Courtney Cazden, Bill Cope, Norman Fairclough, James Gee, Mary Kalantzis, Gunther Kress, Allan Luke, Carmen Luke, Sarah Michaels e Martin Nakata. Esses pesquisadores, provenientes de contextos e culturas diferentes, compartilhavam questionamentos relacionados às novas demandas proporcionadas pelas rápidas e constantes mudanças no mundo e à necessidade de formar sujeitos criadores de significados sociais em seus ambientes de trabalhos, em sua comunidade e em suas vidas. Segundo Kalantzis e Cope (1996/2000, p. 3), o GNL fazia o seguinte questionamento: "O que constitui o ensino apropriado de letramento no contexto de fatores cada vez mais críticos acerca da diversidade local e da conectividade global ${ }^{4}$ ".

Essa reflexão tem como base o fato de que era percebido que as mudanças rápidas nas mídias de comunicação e o crescente intercâmbio cultural começavam a orientar uma mudança radical na forma de conceber a pedagogia dos letramentos. O GNL apontava para o fato de que os jovens estavam começando a fazer uso de ferramentas de acesso à comunicação e à informação, o que acarretava novas formas de dizer sobre o mundo, ou seja, novos letramentos de caráter multimodal e multissemiótico.

Segundo Rojo (2013), o GNL buscava justamente apontar, já de saída, por meio do prefixo "multi", para dois tipos de "múltiplos" que as práticas de letramento contemporâneas envolvem: por um lado, semioses e mídias envolvidas na criação de significação para os textos multimodais contemporâneos e, por outro, a pluralidade e a diversidade cultural trazidas pelos autores/leitores contemporâneos a essa criação de significação.

\footnotetext{
4 "What constitutes appropriate literacy teaching in the context of the ever more critical factors of local diversity and global connectedness?"
} 
No que diz respeito à multiplicidade cultural, o GNL voltava seu pensamento para o enfraquecimento da dicotomia cultura erudita / cultura popular. A maioria das produções que circulam em nossa sociedade por meio de mídias digitais ou impressas são repletas de semioses diversas (sons, imagens estáticas, imagens em movimentos, vídeos etc.) que, adicionadas à escrita e/ou à fala, constroem diferentes modos de dizer sobre o mundo. E essa multimodalidade de textos (KRESS, 2010), a segunda representação do prefixo multi apontado pelo GNL, está conectada às profundas transformações nas relações sociais entre aqueles que produzem e se engajam com o texto. A escolha dos modos para a comunicação segue critérios de escolha pessoal orientados pelos tempos em sua determinada comunidade, caracterizando suas práticas de construção de significados.

Segundo Kalantzis e Cope (1996/2000, p. 6), para o GNL, "lidar com diferenças linguísticas e culturais tornou-se central para a pragmática do nosso trabalho, vida civil e privada". À luz das reflexões previamente apresentadas, o GNL propõe a Pegagogia dos Multiletramentos com o intuito de possibilitar "que os alunos se beneficiem da aprendizagem em formas que lhes permitam participar plenamente na vida comunitária pública e econômica" (KALANTZIS; COPE, 1996/2000, p. 9).

\section{O RECORTE DO CURSO FOCAL}

A pesquisa aqui apresentada está situada em um macroestudo vivenciado durante o primeiro semestre do ano de 2015 na disciplina de Inglês VI do sexto período do curso de Letras-Inglês (Lic.) da UFPE, sob a coordenação da Profa. Dra. Maria Cristina Damianovic, coautora desse texto. Mais especificamente, o foco será na experiência vivida em (2015.1) no ensino de produção escrita, na disciplina de Inglês VI, com base na Pedagogia dos Multiletramentos (KALANTZIS; COPE, 1996/2000; 2009), com foco na multimodalidade (KRESS, 2003; 2010), apresentada aqui em recorte.

Neste artigo, nos voltaremos para a produção escrita de três versões de um multimodal essay produzido pelo aluno ao qual chamaremos de Aluno 1. Segundo nossa análise preliminar, este aluno apresentou mais evidências de expansão da argumentação quando da produção do seu texto.

O gênero essay, que é composto por sequências tipológicas (MARCUSCHI, 2008) descritivas e argumentativas, foi escolhido como o gênero textual a ser trabalhado nessa disciplina por ser uma produção textual comum em trabalhos de final de curso, processos de seleção de bolsas para mobilidade discente internacional, publicações (inter)nacionais, bem como na obtenção de certificados internacionais de competência linguística em língua inglesa.

Por considerarmos de extrema relevância os aspectos interculturais dos gêneros (BHATIA, 1993; 2002), a palavra essay não será traduzida neste estudo. Acreditamos que fazê-lo poderia conduzir o leitor a pensar a produção textual em análise por um prisma que não satisfaz a realidade deste estudo.

Multimodal Essay, segundo Dias (2016b), é um texto de caráter explicativoargumentativo que preserva as características principais de um essay tradicional e que busca, por meio da utilização de imagens, desenhos, fotografias e /ou links para áudio e 
vídeos, comunicar uma ideia de forma clara, assertiva e significativa entre sujeitos contemporâneos comunicadores de significados em uma sociedade imersa em semioses múltiplas.

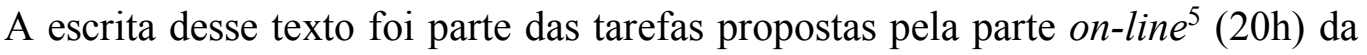
disciplina de Inglês VI, que se deu por meio da plataforma do Edmodo. Essa parte do curso foi nomeada de English VI - Developing Essays in a Multimodal and Multiliterate Community. (Inglês VI - Desenvolvendo Essays em uma Comunidade Multiletrada e Multimodal) (LIMA, 2015). Esse trabalho surge como uma proposta de reforma curricular (DAMIANOVIC, 2015) para o ensino da língua inglesa na turma de sexto período da UFPE, parte presencial (40h) e parte on-line (20h).

Dois professores trabalharam juntos na condução do conteúdo on-line da disciplina e serão chamados aqui de Nelson e Lorena ${ }^{6}$. As três unidades didáticas que compõem o curso foram elaboradas por Lima (2015). O curso online foi conduzido pelos professores citados anteriormente da forma mencionada a seguir: Nelson ficou responsável pela correção de todas as tarefas que orientavam o desenvolvimento da argumentação em textos argumentativos multimodais. Lorena cuidou das devolutivas crítico-colaborativas (DIAS, 2016b) orientadoras da produção escrita dos multimodal essays.

Segundo Dias (2016b), uma devolutiva crítico-colaborativa tem um caráter de formação intervencionista e toma como base a natureza dialógica da linguagem (BAKHTIN, 2008) para promover, por meio da argumentação como processo crítico de negociação (FUGA; LIBERALI, 2014), a possibilidade de reflexão e reformulação crítica de opiniões inicias acerca de uma ideia e (re)posicionamento social.

Portanto, uma devolutiva crítico-colaborativa se afasta do discurso de poder e objetiva promover espaços de expansão dialógica ${ }^{7}$ entre formador e formando, garantindo a possibilidade de negociação de sentidos no movimento de produção do novo.

Lorena também informava Nelson, designer do curso on-line, das áreas da organização argumentativa enunciativa, linguística e discursiva (LIBERALI, 2013) que precisavam ser expandidas nas unidades on-line. Para este artigo, focaremos exclusivamente na importância da multimodalidade para a expansão argumentativa no processo de produção dos textos do aluno focal.

\footnotetext{
${ }^{5} \mathrm{Na}$ experiência vivida na turma de Inglês VI (2015.1), parte do curso foi on-line e parte presencial em sala de aula. Aqui, nós nos voltamos para a parte on-line do curso.

${ }^{6}$ Nomes fictícios escolhidos pelos pesquisadores.

${ }^{7}$ Utilizamos os termos discurso de poder e expansão dialógica com base nos estudos bakhtinianos para explicar que, em uma devolutiva de caráter crítico-colaborativo, o professor (figura de poder) não deve se utilizar de sua posição para impor retificações ao texto do aluno, mas sim convidá-lo ao diálogo se utilizado do principio de que o enunciado é de natureza ativamente- responsiva e dialógica.
} 


\subsection{O GÊNERO ESSAY E SEU DESIGN NO ENGLISH VI- DEVELOPING ESSAYS IN A MULTIMODAL AND MULTILITERATE COMMUNITY}

O Longman Contemporary Dictionary ${ }^{8}$ define essay sob duas perspectivas 1) um pequeno texto escrito por um aluno como parte de um estudo; 2) um pequeno texto que fornece a alguém ideias sobre política, sociedade etc.

Uma definição mais robusta desse gênero textual pode ainda ser encontrada na página on-line do UK Essays ${ }^{9}$, que define essay como um texto que normalmente se baseia no ponto de vista do autor. São textos não ficcionais, mas frequentemente subjetivos. O site ainda aponta que essays podem conter crítica literária, manifestos políticos, argumentos adquiridos, observações de vida cotidiana, lembranças e reflexões do autor. Um essay ainda pode ser caracterizado da seguinte forma: descritivo, narrativo, comparativo e contrativo, persuasivo e argumentativo. No nosso estudo, o interesse está voltado para a expansão da argumentação em textos escritos, e daí a nossa predileção pela escrita e analise de essays de caráter argumentativo, com foco em temas controversos.

Argumentative essays são mais frequentemente utilizados para debater questões controversas, ou seja, questões sérias, sobre as quais existe algum desacordo evidente. Esses textos, portanto, apresentam um argumento principal e em seguinda oferecem razões para validar a sua veracidade.

\subsection{ASPECTOS ARGUMENTATIVOS ENUNCIATIVOS, DISCURSIVOS E LINGUÍSTICOS}

Segundo a Pedagogia dos Multiletramentos, "todas as formas de representação, incluindo a linguagem, deveriam ser consideradas como um processo dinâmico de transformação ao invés de processos de reprodução" (KALANTZIS; COPE, 2009, p. 175). As atividades do referido curso on-line, que orientaram a produção textual dos alunos, tinham como objetivo principal oferecê-los a oportunidade de escrever utilizando os diferentes modos de composição do texto contemporâneo e experienciar como essas semioses diversas expandem a argumentação na escrita do gênero textual supracitado. Esse fenômeno será analisado à luz de algumas das categorias argumentativas enunciativas, discursivas e linguísticas em Liberali (2013). Por isso, para a análise de dados deste artigo, descreveremos somente os aspectos argumentativos que usaremos para a análise dos dados em recorte para este estudo.

Consoante à autora supracitada, as características enunciativas da argumentação focalizam o contexto em que o evento é realizado, tendo como base a dialética entre o local, o momento, veículo, participantes desse evento, objetivos finais e conteúdos debatidos e seus modos concretos de produção e realização.

Dessa forma, o foco argumentativo enunciativo recai, no nosso caso, sobre: a) local e momento de produção (Disciplina de Inglês VI na graduação em Letras - Língua Inglesa (Lic.) curso on-line); b) papel dos interlocutores (alunos da graduação em Letras - Língua Inglesa (Lic.) escrevendo um multimodal essay para a referida disciplina); c)

\footnotetext{
${ }^{8}$ Acesso disponível em: http://www.ldoceonline.com/dictionary/essay.

${ }^{9}$ https://www.ukessays.com/resources/help-guides/undergraduate/essay/what-is-an-essay.php.
} 
objetivos da interação-fim (atender às regras norteadoras para a escrita do texto em questão: as devolutivas crítico-colaborativas, para escrever um multimodal essay que visa colaborar para a construção do pluralismo (LIBERALI, 2013, p. 65) de opiniões sobre o tema "liberdade de expressão"); e d) objeto/conteúdo temático (multimodal essay sobre tema "liberdade de expressão" com sua devida organização enunciativa, discursiva e linguística). Para este estudo, nos voltaremos apenas para a análise do aspecto relacionado ao objetivo da Interação-fim.

Os aspectos argumentativos discursivos compreendem o modo como o texto é disposto em relação a: i) plano organizacional, ii) organização temática, iii) foco sequencial e iv) articulação entre as ideias apresentadas. Para este estudo, nos voltaremos apenas para a disposição textual no que diz respeito ao plano organizacional.

Em relação às características linguísticas da argumentação, serão analisados os mecanismos de valoração, que marcam a posição do locutor frente aos temas, aos interlocutores, ao momento de enunciação etc. Geralmente são expressos por adjetivações, expressões depreciativas ou apreciativas, expressões descritivas e identificatórias.

O nosso estudo contará também com o uso e o conceito da palavra design ${ }^{10}$, que, para os Multiletramentos, tem um significado muito forte e está diretamente associado ao poder de produção de significados de um indivíduo. Design, "no que diz respeito à construção, é aquilo que você faz no processo de representação e significados para alguém no processo de produção de sentido, como na leitura, escuta, visualização, ou para o mundo em processos comunicativos como a escrita, fala ou criação de imagens" (KALANTZIS; COPE, 2009, p. 175). Segundo a Pedagogia dos Multiletramentos, o conceito de design possui três aspectos:

1) Available design ${ }^{11}$ (design disponível) - Formas de representação já existentes: contextos e convenções já existentes em uma cultura para produzir significado.

2) Designing - $\mathrm{O}$ ato, em si, de construir. O trabalho que alguém faz quando está construindo significados. $O$ processo pelo qual alguém se apropria e transforma e dá uma nova voz aos designs já existentes;

3) The redesigned - $\mathrm{O}$ produto do processo pelo qual, por meio do designing, o mundo e o indivíduo são modificados.

O nosso objetivo, com este estudo, é analisar a expansão argumentativa escrita do Aluno 1 durante o processo de designing do gênero essay (que deu origem aos multimodal essays) segundo o recorte das categorias argumentativas em Liberali (2013) apresentadas anteriormente.

\section{DISCUSSÃO DOS DADOS}

Após discussão sobre as características e relevância da utilização real do gênero essay (available design) em situações acadêmicas para ambos professores e estudantes de

\footnotetext{
${ }^{10}$ Mantivemos a palavra design em inglês, uma vez que a mesma é costumeiramente usada no meio acadêmico atual.

${ }^{11}$ Todos os termos que explicam a Pedagogia dos Multiletramentos serão mantidos em inglês.
} 
LI, os alunos focais foram orientados a escrever um essay com o seguinte tema: In a democratic society, every citizen should have the right to express any of their thoughts freely ${ }^{12}$.

Segundo as instruções do professor Nelson, os alunos deveriam seguir o plano organizacional tradicional do available design (apresentação do argumento, suporte ao argumento, contra-argumento, suporte ao contra-argumento e síntese) para escreverem seus textos, bem como adicionar pelo menos um suporte multimodal em qualquer parte de sua produção. A sequência de designing dos multimodal essays seguiu a seguinte ordem.

Figura 1 - Fluxo de designing.

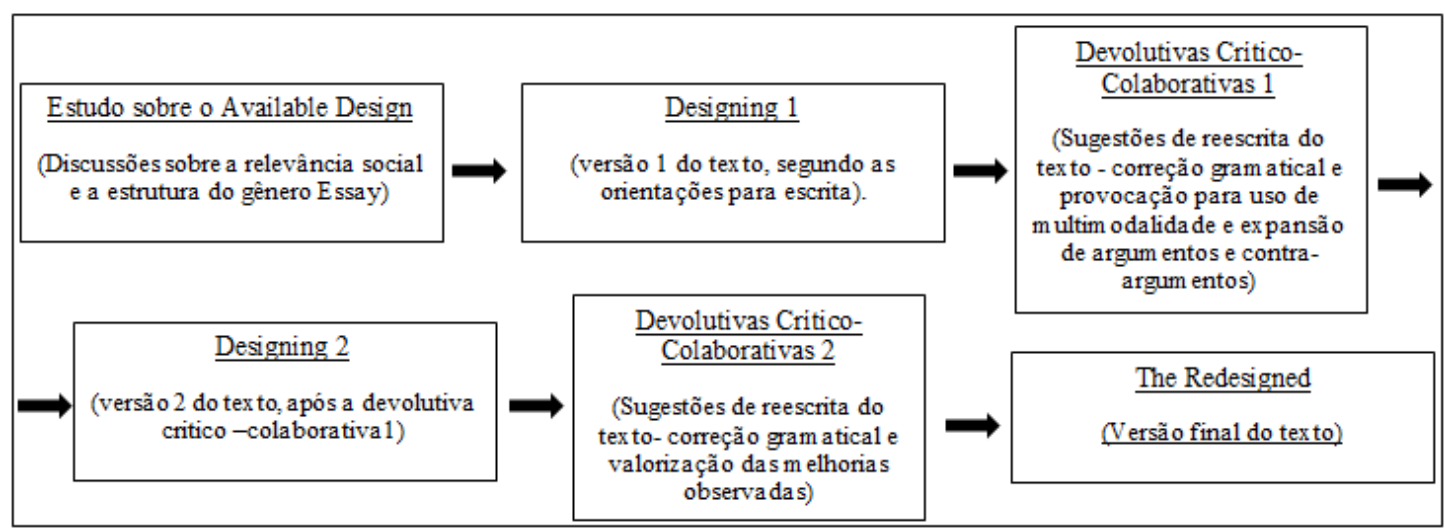

Fonte: Produzido pelas autoras.

Segundo a Pedagogia dos Multiletramentos, o ato de designing está relacionado à possibilidade de comunicar significados que representem subjetivamente o mundo com base em designs já disponíveis (available designs). O ato de criar seria, dessa forma, um ato empoderador da capacidade cognitiva do sujeito, uma vez que, diferentemente dos métodos mais tradicionais de letramento, o potencial criador subjetivo é reconhecido e levado em consideração nesse processo.

Os autores afirmam:

aquilo que o criador de significados cria é um novo design, uma expressão de sua voz que toma como base a mistura única de recursos, códigos e convenções para criação de significados que se encontram disponíveis em seu contexto e cultura. O momento de designing é um momento de transformação, de recriação de mundo por meio da representação do mundo novo. ${ }^{13}$ (KALANTZIS; COPE, 2009, p. 177)

\footnotetext{
12 Numa sociedade democrática, todos os cidadãos devem ter o direito de expressar livremente qualquer um dos seus pensamentos. Esse tema foi escolhido porque, na época (2015.1), havia um debate efervescente em relação ao atentado terrorista ao jornal Francês Chalie Hebdo e a existência ou não do limite para a liberdade de expressão.

13 "What the meaning maker creates is a new design, an expression of their voice which draws upon the unique mix of meaning-making resources, the codes and conventions they happen to have found in their contexts and cultures. The moment of design is a moment of transformation, of remaking the world by representing the world afresh."
} 
Tomando como base a diversidade de modos como os significados são encontrados nas atividades vividas pelos sujeitos na vida contemporânea (LIBERALI et $a l ., 2016)$ e o poder subjetivo do ato de criação de significados, nos voltaremos para a análise da expansão argumentativa quando do processo de designing de um multimodal essay à luz das categorias argumentativas citadas anteriormente para o recorte dos dados em questão neste estudo. forma:

A primeira produção textual (Designing 1) do Aluno 1 se caracteriza da seguinte

A) Aspectos Argumentativos Enunciativos - (categoria escolhida: Objetivos da Interação-Fim). Os alunos tinham como objetivo argumentar sobre a liberdade de expressão por meio de um multimodal essay. Para que esse objetivo fosse alcançado, era necessário que, além de utilizar e expor suas ideias e dar suporte adequado às mesmas, segundo o plano organizacional de um essay tradicional, era salutar adicionar, pelo menos, um elemento multimodal em consonância com alguma parte do texto.

Assim como pode ser visto no Anexo 1, o aluno optou por não utilizar suporte multimodal em parte alguma do seu texto. O que não comprometeu o objetivo de argumentar sobre seu ponto de vista em relação ao tema em questão. O resultado do Designing 1 é, sob esse aspecto, um essay monomodal de caráter tradicional, que comunicou de forma satisfatória as ideias do Aluno 1 sobre o tema Liberdade de Expressão.

B) Aspectos Argumentativos Discursivos - (categoria escolhida: Plano Organizacional). Os alunos precisavam iniciar o texto expondo seu argumento e dando-lhe suporte logo em seguida. Era necessário também que um ponto de vista controverso fosse apresentado e sustentado para que conflito das ideias opostas resultasse em uma síntese coerente com o debate das ideias apresentadas.

A estrutura do plano organizacional foi seguida de forma satisfatória pelo Aluno 1, mas observou-se ainda espaço para desenvolvimento dos argumentos e seus suportes. Observou-se o cuidado em atender à estrutura organizacional do texto, mas percebe-se que as ideias expostas pelo Aluno 1 ainda poderiam ser expandidas por meio de explicações e exemplificação de conceitos citados ao longo dos parágrafos, como se observa no excerto do Designing 1 do Aluno 1 a seguir, no qual o aluno mencionou o conceito de censura ditatorial (dictatorial censorship) mas não o exemplificou com fatos em outras partes do texto.

Figura 2 - Parágrafo1 - Designing 1.

Around the world there are people who think that speaking their minds is right. We have come a long way, from dictatorial censorship, and fear of retaliation, not to use this acquired right as often as we please.

Fonte: Cedido pelo Aluno. 
C) Aspectos Argumentativos Linguísticos - (categoria escolhida: mecanismos de valoração). Segundo a definição utilizada anteriormente nesse estudo, um essay é um texto de caráter explicativo-argumentativo que tem como objetivo expor um ponto de vista. Por isso, acreditamos que as escolhas lexicais feitas pelo autor desse tipo de texto são essenciais para validar a sua posição frente ao tema em questão.

Em sua produção inicial, o Aluno 1 fez uso de adjetivações, advérbios de intensidade e expressões apreciativas e depreciativas, assim como esperado para o tipo de texto que escreveu. A seguir, um excerto mostra a utilização de expressões depreciativas usadas para dar suporte a um argumento exposto pelo Aluno 1. A escolha dessas expressões demonstra a preocupação do aluno em relação ao uso distorcido do conceito de liberdade de expressão. A figura abaixo mostra a parte do texto do Aluno 1 sobre a qual estamos nos referindo:

Figura 3 - Parágrafo 4 - Designing 1.

One thing is someone having a different opinion from you, as for example, not liking certain kind of music. But from the moment said person says that that specific brand of music is made only by criminals, for instance, they are incurring in a crime of defamation. Hundreds of racist, homophobic, xenophobic, islamophobic and so forth, speeches are disguised as "Freedom of Speech" every day.

Fonte: Cedido pelo Aluno.

A segunda produção textual do Aluno 1 foi orientada por uma intervenção de caráter formativo da professora de nome fictício Lorena, que utilizou como estratégia as devolutivas crítico-colaborativas definidas anteriormente neste artigo.

O objetivo principal dessas orientações de reescrita era possibilitar que os alunos focais pudessem ser agentes fazedores e refazedores de sentindo (LIBERALI, 2016) por meio de instrumentos multimodais utilizados cotidianamente. Por isso, as intervenções tiveram caráter formativo para além da correção de possíveis incoerências gramaticais no texto. Distanciando-se de uma postura que considerava tradicional e recorrente em suas intervenções enquanto profissional, a professora optou por não utilizar o discurso monossêmico e rígido no qual prepondera a autoridade, mas sim deixar clara a intencionalidade de convite à reflexão e composição de possibilidades (LIBERALI, 2016) para a reescrita dos textos. (Ver DL1 ${ }^{14}$ DL2 ${ }^{15}$ ).

\footnotetext{
14 Tradução para a língua portuguesa: Seu argumento foi apresentado com clareza. Como você acha que poderia acrescentar um suporte multimodal nessa parte do seu texto?

${ }^{15}$ Tradução para a língua portuguesa: Que tal usar um link para um website a fim de explicar o significado deste conceito?
} 
Figura 4 - Devolutivas crítico-colaborativas ao parágrafo 1 do Design 1.

Around the world there are people) who think that speaking their minds is
right. We have come a long way, from dictatorial censorship]. and fear of -... [DL1] Comentário: Your argument
retaliation, not to use this acquired right as often as we please.

Fonte: Cedido pelo Aluno.

A segunda produção textual (Designing 2) do Aluno 1, após o contato com as devolutivas crítico-colaborativas do professor, apresenta as seguintes caraterísticas, conforme o Anexo 2:

A) Aspectos argumentativos enunciativos - (categoria escolhida: objetivos da interação-fim): A adição de links para websites, bem como a utilização de imagens para expandir e exemplificar argumentos, além de adicionar densidade discursiva e veracidade ao texto, atende ao objetivo da interação-fim e fazem surgir o que definimos nesse estudo como multimodal essay. O momento de Designing 2 exemplifica claramente uma situação em que houve espaço para o surgimento de objetivos compartilhados entre formador e formando, visto que a intencionalidade da expansão dialógica ${ }^{16}$ (BAKHTIN, 2008) presente na enunciação do professor convida o aluno a refletir sobre a sua produção e responder criticamente à mesma. Acreditamos, assim, que o caráter formativo da intervenção do professor, por meio de suas Devolutivas Crítico-Colaborativas, foi de extrema importância para que houvesse expansão argumentativa enunciativa nesse momento de produção textual do Aluno 1.

B) Aspectos argumentativos discursivos - (categoria escolhida: plano organizacional): o Design 2 conservou a sequência estrutural da primeira versão (argumento, suporte, contra-argumento, suporte e síntese). Todavia, a adição de suporte multimodal possibilitou que as ideias usadas na composição de argumentos e contra-argumentos, bem como seus suportes, fossem explicadas e esclarecidas, possibilitando a expansão da argumentação ao longo de todo o multimodal essay. Como resultado da expansão dessas ideias e argumentos, o número de parágrafos do texto aumentou. (Ver anexo 3).

\footnotetext{
16 Nessa perspectiva, a expansão dialógica está relacionada ao fato de o professor, enunciador das devolutivas crítico-colaborativas, possibilitar que o aluno possa ser também agente na ação de refletir sobre a reconstrução do seu texto. O diálogo ganha expansão porque o professor, ao fazer perguntas ao aluno , deixa de ser o centro da interlocução.
} 
Figura 5 - Designing 2 (multimodal essay) - Adição de imagens e links ao texto original.

Around the world there are people who think that speaking their minds is right. We have come a long way, from dictatorial censorship, and fear of retaliation, not to use this acquired right as often as we please. Most countries in the world has suffered from some kind of dictatorship and now refluses to go back to this era of darkness.

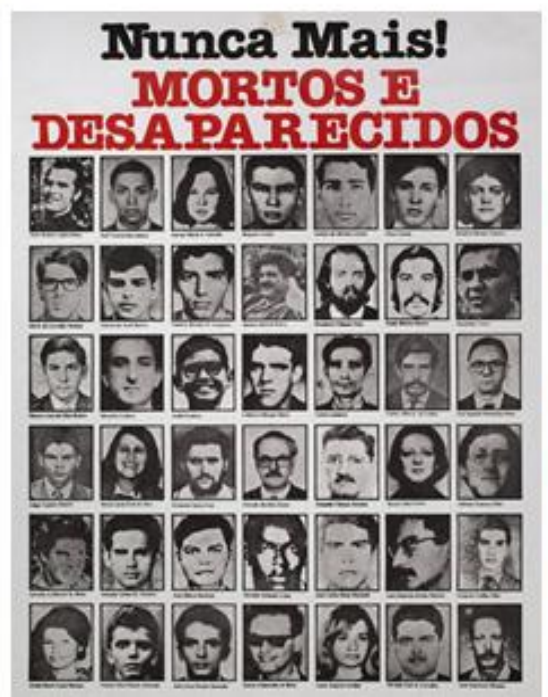

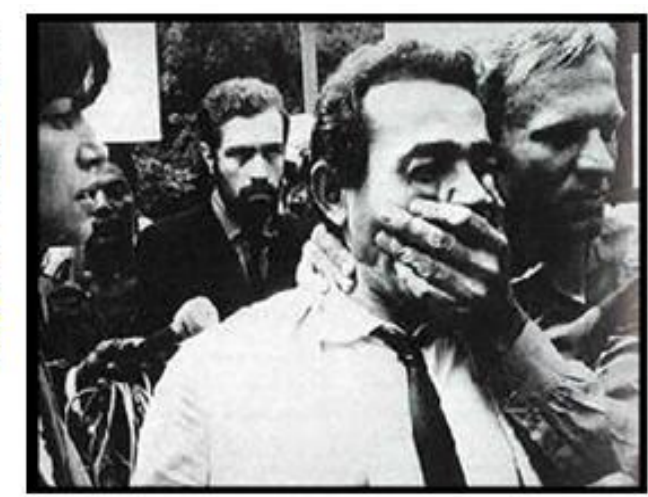

Take Brazil for example, for about twenty years many people were tortured, kidnapped and even killed for speaking something that went even slightly against the government.

Freedom of speech is known and used expression that refers to our right of saying what is on our minds. Many hundreds people fought for it through different periods of time, which culminated in the Article 19 of the Universal Declaration of Human Rights. It gives every human being a free voice and the liberty to use it.

Fonte: Cedido pelo Aluno.

C) Aspectos Argumentativos Linguísticos - (categoria escolhida: mecanismos de valoração): a reelaboração discursiva do texto em vários momentos de seu plano sequencial, orientada pela acomodação de suportes multimodais para expansão de ideias e argumentos, materializou-se por meio de novas escolhas lexicais para dizer sobre essas ideias.

Novamente destacamos o uso de adjetivação para fundamentar a posição do Aluno 1 em relação ao argumento exposto, bem como o uso de advérbios e expressões apreciativas e depreciativas. Contudo, a utilização desses recursos linguísticos ocupou posição de mais destaque na construção de significado ao longo do multimodal essay, como mostra o quadro a seguir, que destaca os mecanismos de valoração que marcam mais assertivamente a posição do autor em relação aos pontos de vistas expostos. 
Quadro 1 - Aspectos Argumentativos Linguísticos - (mecanismos de valoração) - Designing 2.

\begin{tabular}{|c|l|}
\hline Parágrafo & \multicolumn{1}{|c|}{$\begin{array}{c}\text { Mecanismos de valoração (adjetivos, advérbios, expressões valorativas e } \\
\text { depreciativas) }\end{array}$} \\
\hline 1 & $\begin{array}{l}\text { Era of darkness - Usado para adjetivar, por meio de valor negativo, o período de } \\
\text { ditadura vivido por alguns países. }\end{array}$ \\
\hline 2 & $\begin{array}{l}\text { Slightly against - Usado exemplificar a seriedade das consequências sofridas por } \\
\text { qualquer individuo que fosse, até de forma leve, contrário às opiniões do governo } \\
\text { brasileiro em tempos de ditadura. }\end{array}$ \\
\hline 3 & $\begin{array}{l}\text { Every human being - Expressão utilizada para intensificar os direitos concedidos a } \\
\text { todo e qualquer ser humano pelo artigo 19 da Declaração Universal dos Diretos } \\
\text { Humanos. }\end{array}$ \\
\hline 5 & $\begin{array}{l}\text { All - Utilizado também no Design 1 para informar que é possível encontrar exemplos } \\
\text { de abuso do direito a liberdade de expressão em toda e qualquer parte do mundo. }\end{array}$ \\
\hline 5 & $\begin{array}{l}\text { Mantiveram-se as expressões depreciativas usadas no Design 1 para exemplificar } \\
\text { crimes cometidos em nome da liberdade de expressão (racist, homophobic, } \\
\text { xenophobic, islamophobic). Todavia como o parágrafo cresceu com o intuito de } \\
\text { expandir a argumentação por meio de exemplificação, adjetivações depreciativas } \\
\text { (extremist, radicals) foram usadas para caracterizar agentes dos crimes citados } \\
\text { anteriormente. }\end{array}$ \\
\hline 6 & $\begin{array}{l}\text { Internalized prejudice, ignorant, malicious - Adjetivações utilizadas no parágrafo } \\
\text { final (síntese) para descrever pessoas que usam a liberdade de expressão para cometer } \\
\text { crimes contra a humanidade. O uso dessas expressões marca claramente a posição de } \\
\text { repúdio do Aluno 1 a esse tipo de atitude. }\end{array}$ \\
\hline
\end{tabular}

Fonte: Produzido pelas autoras.

Segundo a Pedagogia dos Multiletramentos, àquilo que, de resíduo, de traços de transformações é deixado no mundo social chama-se de The Redesigned. "Os textos do designing se transformam em The Redesigned, novas formas para significar no jogo aberto e dinâmico da subjetividade e significados" (KALANTZIS; COPE, 2009, p. 177).

A terceira produção textual do Aluno 1 será chamada de The Redesign, uma vez que representa o produto final do processo de Designing, que contou com duas sessões de Devolutivas Crítico-Colaborativas e duas oportunidades de reescrita do texto original.

O multimodal essay final, ou seja, The Redesigned, se caracteriza por conservar os aspectos expandidos na versão anterior do texto e por revelar algumas modificações consequentes às respostas à segunda sessão de Devolutivas Crítico-Colaborativas.

Uma delas está relacionada ao aspecto argumentativo discursivo, uma vez que o plano sequencial foi alterado em tamanho (adição de mais informações a um dos parágrafos) para acomodar uma sugestão apontada durante a segunda sessão de Devolutiva Crítico-Colaborativa, como mostra a figura abaixo. (Ver DL3 ${ }^{17}$ ).

\footnotetext{
${ }^{17}$ Maravilhosa escolha de imagens. Que tal explorar a imagem deste parágrafo um pouco mais? Tente trazer a informação por trás dela para o texto. Você apresentou um argumento muito bom neste parágrafo. Vamos torná-lo ainda melhor? Todas as traduções foram feitas pela pesquisadora Leandra Dias.
} 
Figura 6 - Devolutivas Crítico-Colaborativas ao parágrafo 2 do Designing 2.

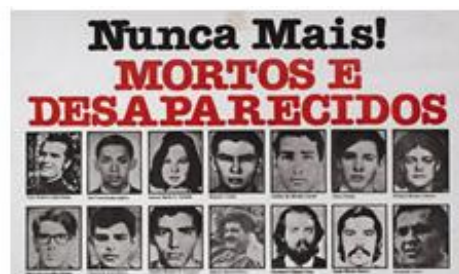

\section{IIrF}

Take Brazill for example, for about twenty years many people were tortured kidnapped and even killed for speaking something that went even slightly against the government.

\section{Fonte: Cedido pelo Aluno.}

Em resposta à sugestão feita na devolutiva crítico-colaborativa acima, o Aluno 1 acrescentou informações ao parágrafo mostrado na Figura 6, de modo a estabelecer maior relação de sentido com a figura da esquerda, que mostra a foto de pessoas desaparecidas e mortas durante o período da Ditadura Militar. Para tal, o Aluno 1 fez menção direta à figura mencionada anteriormente, indicando sua posição em relação a parágrafo e estabelecendo conexão entre o leitor e os rostos das pessoas lá expostas. Observamos aqui outro nível de evolução do uso intercalado entre multimodalidade e argumentação: a menção direta ao suporte multimodal para validar e dar suporte a um argumento apresentado anteriormente no texto.

Consequentemente, o aspecto argumentativo enunciativo também foi expandido, uma vez que vemos, também nessa reelaboração textual, a preocupação em atender ao objetivo de interação-fim, que era escrever um multimodal essay e comunicando assertivamente suas ideias. Não encontramos, todavia, uso significativo de expressões valorativas, e, por isso, não consideramos que houve expansão no aspecto argumentativo linguístico nesse momento da produção textual.

Figura 7 - Parágrafo anterior modificado - The Redesigned.
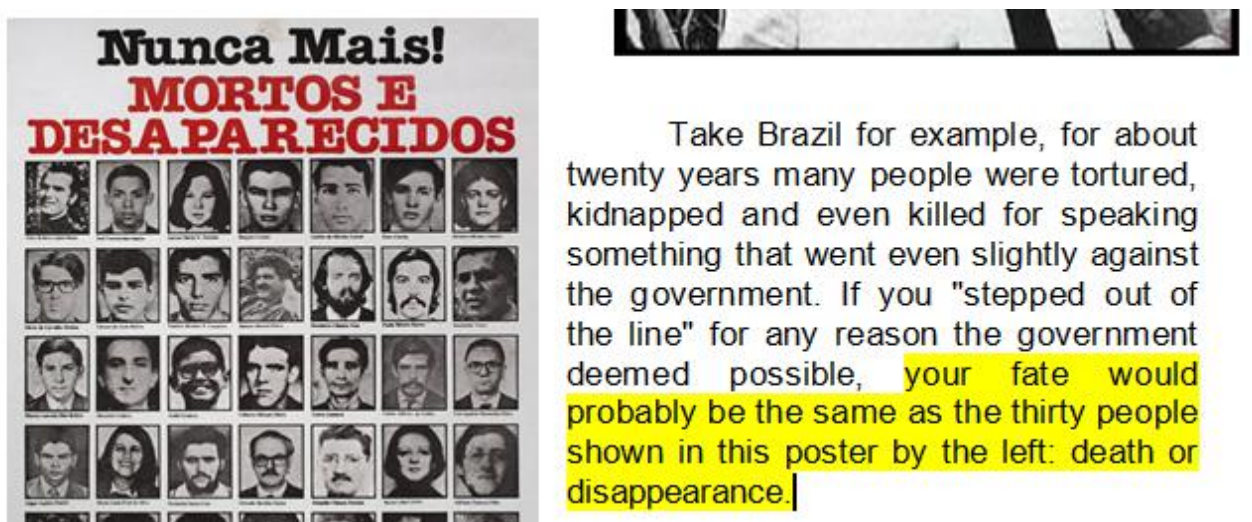

Take Brazil for example, for about twenty years many people were tortured, kidnapped and even killed for speaking something that went even slightly against the government. If you "stepped out of the line" for any reason the government deemed possible, your fate would probably be the same as the thirty people shown in this poster by the left: death or disappearance.|

Fonte: Cedido pelo Aluno.

A versão final do multimodal essay produzido pelo Aluno 1, a qual chamamos nesse estudo de The Redesigned, segundo a Pedagogia dos Multiletramentos, pode ser encontrada na íntegra no Anexo 3. 


\section{CONSIDERAÇÕES FINAIS}

A questão que tínhamos como objetivo tratar, neste artigo, era a articulação entre argumentação e multimodalidade (LIBERALI, 2016) na produção escrita na graduação de Letras-Língua Inglesa (Lic.). A justificativa do nosso interesse nessa pesquisa tem fundamentação no fato de que, apesar de as escolhas multissemióticas já serem consideradas parte inerente das estratégias de comunicação daqueles que produzem e se engajam com o texto contemporâneo, esse assunto ainda necessita ser abordado como prática comunicativa dentro e fora da escola. E foi na tentativa de atender a essa demanda que surgiu a pesquisa que deu origem a esse estudo. Para isso, foi oferecido um curso online de 20h (LIMA, 2015) para um grupo de alunos do sexto período da graduação de Letras-Língua Inglesa (Lic.) da UFPE, com o objetivo de fazê-los experienciar de forma didatizada a articulação entre argumentação e multimodalidade por meio da escrita de um multimodal essay.

A análise da produção textual do aluno focal em suas três versões, com base na visão de design da Pedagogia dos Multiletramentos, chama a atenção para o fato de que, apesar de sermos seres sociais integrantes de uma sociedade multissemiotizada, ainda interagimos de forma reticente com a inserção dessa multiplicidade de modos na construção de significados em contextos escolares. A exemplo disso, temos a primeira produção do Aluno 1, que, apesar das instruções sobre a adição de suporte multimodal, caracterizou-se pela preservação das características tradicionais do gênero textual em questão.

Observamos ainda que a intervenção do professor por meio das devolutivas crítico-colaborativas (DIAS, 2016b) foi de relevância singular, uma vez que, com essas intervenções, o Aluno 1 conseguiu ter acesso a uma experiência de aprendizagem e criação de significado de caráter reflexivo. Com isso, esse aluno se colocou longe da experiência tradicional do design autoritário, ou seja, a imposição da escolha de um padrão pré-determinado no processo de criação. Assim, entrou em consonância com a Pedagogia dos Multiletramentos, que concebe o momento de designing como um momento de transformação e de reestruturação daquilo que já se encontra disponível como design no mundo para criação do novo.

Identificamos ainda que a adição e a acomodação de suporte multimodal ao gênero argumentativo ensaio(essa) durante o processo de designing, que resultou naquilo que viemos a chamar de multimodal essay (DIAS, 2016a), teve impacto bastante significativo na expansão dos aspectos argumentativos enunciativos, discursivos e linguísticos observados neste estudo quando da comparação da versão 1 do texto com a sua versão 3 , aqui chamada de The Redesigned.

A escolha multimodal do Aluno 1 se deu por meio de imagens jornalísticas para exemplificar fatos validando argumentos, bem como por meio do uso de hyperlinks para explicar conceitos importantes para o entendimento dos argumentos apresentados. Acreditamos que essas escolhas estão intimamente ligadas ao contrato social ${ }^{18}$ (KRESS, 2012) que orienta a escolha de modos durante a construção de significados, uma vez que

18 Esta entrevista é parte da lição 1 da unidade 1 do curso sobre Multimodalidade disponível no website MODE (Multiliteracies Methodologies) do Instituto de Educação da Universidade de Londres. 
tanto as imagens quanto os hyperlinks escolhidos caracterizam formas de representação socialmente aceitas e utilizadas no contexto enunciativo no qual o texto foi concebido.

Os estudos teóricos sobre argumentação e multimodalidade ainda representam linhas teóricas paralelas. Todavia, acreditamos que a articulação entre os dois campos teóricos pode resultar em grandes contribuições para a compreensão de questões resultantes da natureza comunicativa rica em modos do homem contemporâneo.

\section{REFERÊNCIAS}

BAKHTIN, M. Estética da criação verbal. $6^{a}$. ed. São Paulo: Martins Fontes, 2008.

BHATIA, V.K. Applied genre analysis: a multi perspective model. City University of Hong Kong, Ibérica, 4, p. 3-19, 2002. 1993.

Analysing genre: language use in professional settings. New York: Routledge,

BEZEMER, J.; KRESS, G. Changing text: a social semiotic analysis of textbooks. Designs for Learning, 3 (1-2), p. 10-29. Dezembro, 2010.

COPE, B.; KALANTZIS, M. Introduction: Multiliteracies: the beginnings of an idea. In: COPE, B.; KALANTZIS, M. Multiliteracies: literacy learning and the design of social futures. New York. Cambridge, 1996/2000.

DAMIANOVIC, M.C. Relatório de análise de ementas de Língua Inglesa I-VIII realizado para o Projeto de Reconstrução Curricular da Licenciatura em Letras da UFPE. Comunicação apresentada no VI SIAC. Simpósio de Ação Cidadã. Pontifícia Universidade Católica de São Paulo, novembro de 2015.

DIAS, L.M.S. O entreLACE de (multi) modalidades na produção de "Essays" na graduação de Letras-Inglês. Pôster apresentado no LACE. PUC-SP. 2016 a.

Feedback argumentativo: ressignificando o diálogo entre professor e aluno na correção de textos escritos. Trabalho de final do curso. Teorias da Enunciação [curso ministrado pela professora Dra. Siane Goes no PPG/UFPE], 2016b.

FUGA, V.P; LIBERALI, F.C. Argumentação na atividade de formação crítica, colaborativa e criativa. Em: MATEUS, E; OLIVEIRA, N.B. Estudos críticos da linguagem e formação de professores/as de línguas: contribuições teóricometodológicas. Campinas: Pontes, 2014. p. 49-78.

KALANTZIS, M.; COPE, B. Multiliteracies: new literacies, new learning. Pedagogies [an International Journal], n. 4, p. 164-195, 2009.

Multiliteracies: literacy learning and the design of social futures. New York: Routledge: 2000. 
KRESS, G. Multimodality: key concepts in multimodality. Institute of Education (UCL), 2012. Disponível em: https://mode.ioe.ac.uk/2012/02/16/video-resource-key-conceptsin-multimodality/. Acesso em 27 abri.2018.

Literacy in the New Media Age. Nova York: Routledge, 2003.

Multimodality: a social semiotic approach to contemporary communication. Londres; Nova York: Routledge, 2010.

LIBERALI, F. C. Argumentação em Contexto Escolar. Campinas: Pontes, 2013.

LIBERALI, F.C.; DAMIANOVIC, M.C.; NININ, M.O.G.; MATEUS, E.; GUERRA, M. Argumentação em Contexto Escolar: relatos de Pesquisa. Campinas: Pontes, 2016.

LIMA, N. English VI: developing essays in a multimodal and multiliterate community. Material didático elaborado para a disciplina de Inglês VI, Letras. UFPE, Recife, 2015. Disponível em: https://www.edmodo.com/home\#/group?id=12787977. Acesso em 25 mar. 2017.

MARCUSCHI, L.A. Produção textual, análise de gêneros e compreensão. São Paulo: Parábola Editorial, 2008.

MOITA LOPES, L.P. Fotografias da Linguística aplicada brasileira na modernidade recente: contextos escolares Em: . (Org.). Linguística Aplicada na Modernidade Recente: Festschrift para Antonieta Celani. São Paulo: Parábola, 2013.

ROJO, R. Gêneros discursivos do círculo de Bahktin e Multiletramentos. Em: ROJO, R. (Org.). Escola conectada: os multiletramentos e as TICs. São Paulo: Parábola, 2013.

Pedagogia dos multiletramentos: diversidade cultural e linguagens na escola. Em: ROJO, R.; MOURA, E. (Orgs.). Multiletramentos na escola. São Paulo. Parábola, 2012.

SILVA, K.A.; SANTOS, L.I.S.; JUSTINA, O.D. Entrevista com Kanavillil Rajagopalan: ponderações sobre linguística aplicada, política linguística e ensino-aprendizagem. Revista de Letras Norte@mentos - Revista de Estudos Linguísticos e Literários. Edição 08, Estudos Linguísticos 2011/02. Disponível em: http://projetos.unematnet.br/revistas eletronicas/index.php/norteamento. Acesso em 25 abr. 2018.

Artigo recebido em: julho de 2017.

Aprovado e revisado em: janeiro de 2018.

Publicado em: maio de 2018. 


\section{Para citar este texto:}

DIAS, Leandra; DAMIANOVIC, Maria Cristina. A expansão argumentativa na escrita de Multimodal Essays na graduação em Letras Língua Inglesa. Entremeios [Revista de Estudos do Discurso, ISSN 2179-3514, on-line, www.entremeios.inf.br], Seção Estudos, Programa de Pós-Graduação em Ciências da Linguagem (PPGCL), Universidade do Vale do Sapucaí (UNIVÁS), Pouso Alegre (MG), vol. 16, p. 97-120, jan. - jun. 2018.

DOI: http://dx.doi.org/10.20337/ISSN2179-3514revistaENTREMEIOSvol16pagina97a120 


\section{ANEXO 1 \\ UNIVERSIDADE FEDERAL DE PERNAMBUCO \\ CENTRO DE ARTES E COMUNICAÇÃO \\ DEPARTAMENTO DE LETRAS \\ INGLÊS VI}
In a democratic society, every citizen should have the right to express any of their thoughts freely.

Around the world there are people who think that speaking their minds is right. We have come a long way, from dictatorial censorship, and fear of retaliation, not to use this acquired right as often as we please.

Freedom of speech is a largely known and used expression to refer to our right of saying what is on our minds. Many a hundred people fought for it in through different periods of time, that culminates in the Article 19 of the Universal Declaration of Human Rights $^{1}$.

But to what extent is this valid? All around the world we can observe people abusing this right to hide Hate Speeches. Every citizen have the right to express any of their thoughts. However this does not mean they have a free pass concerning what is right or wrong. You are responsible for your words. And if your words hurt, offend, slander or vilifies someone, you also have the right to pay for what you have said.

One thing is someone having a different opinion from you, as for example, not liking certain kind of music. But from the moment said person says that that specific brand of music is made only by criminals, for instance, they are incurring in a crime of defamation. Hundreds of racist, homophobic, xenophobic, islamophobic and so forth, speeches are disguised as "Freedom of Speech" every day.

So yes. Every citizen should have the right to express any of their thoughts freely, and have the right and the duty to shoulder the repercussion of what they said, also. As for the rest of us, we'll keep watching those whom use their "right" as a tool for exposing their internalized prejudice. Makes easier for us to distant ourselves from them if we want. 


\author{
ANEXO 2 \\ UNIVERSIDADE FEDERAL DE PERNAMBUCO \\ CENTRO DE ARTES E COMUNICAÇÃO \\ DEPARTAMENTO DE LETRAS \\ INGLÊS VI
}

In a democratic society, every citizen should have the right to express any of their thoughts freely

Around the world there are people who think that speaking their minds is right. We have come a long way, from dictatorial censorship, and fear of retaliation, not to use this acquired right as often as we please. Most countries in the world has suffered from some kind of dictatorship and now refuses to go back to this era of darkness.

Take Brazil for example, for about

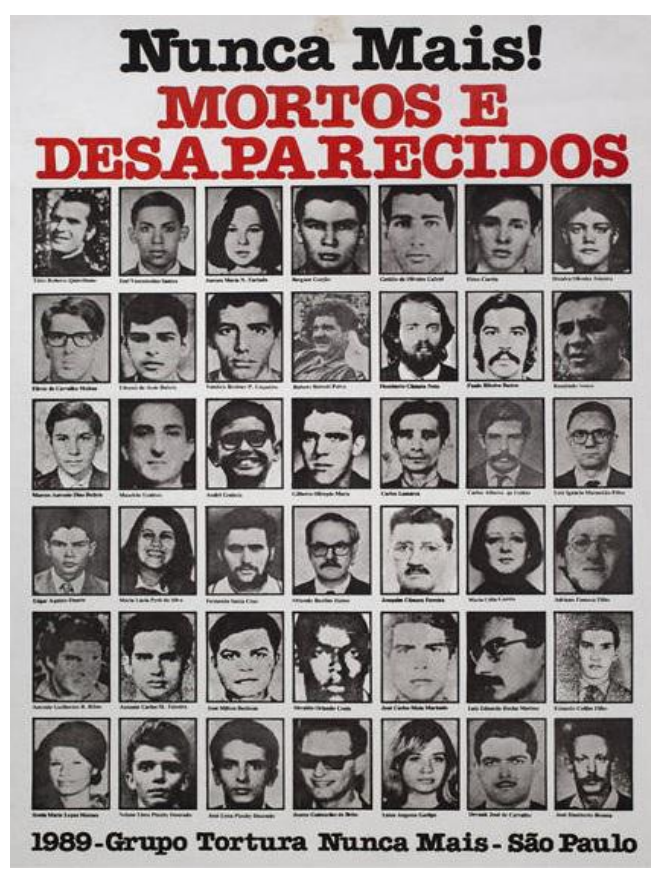

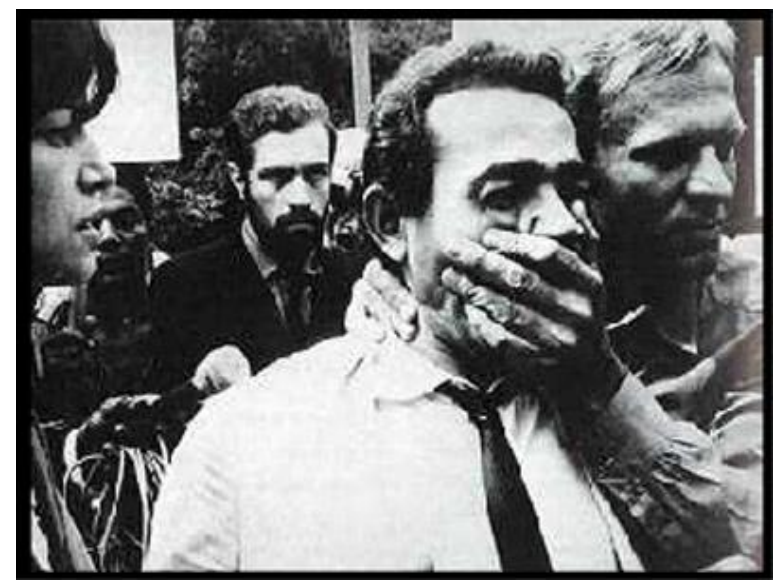

twenty years many people were tortured, kidnapped and even killed for speaking something that went even slightly against the government.

Freedom of speech is known and used expression that refers to our right of saying what is on our minds. Many hundreds people fought for it through different periods of time, which culminated in the Article 19 of the Universal Declaration of Human Rights. It gives every human being a free voice and the liberty to use it.

But to what extent is this valid? All around the world we can observe people abusing this right to hide Hate Speeches. Every citizen has the right to express any of their thoughts.

However this does not mean they have a free pass concerning what is right or wrong. You are responsible for your words. And if your words hurt, offend, slander or vilifies someone, you also have the right to pay for what you have said.

One thing is someone having a different opinion from you, as for example, not liking certain kind of music. But from the moment a person says that that specific brand of music is made only by criminals, for instance, they are incurring in a crime of defamation. Hundreds of racist, homophobic, xenophobic, islamophobic and so forth, speeches are disguised as "Freedom of Speech" every day. A recent example is Europe's 
outbreak of hostility against Islam religion, inciting fear and hate against innocent muslins, all because of extremist attacks made by radicals. Even Hollywood helps perpetrate this stigma by making a blockbuster about a sniper (which according to his own biography enjoyed killing muslins) turned American hero.

So yes. Every citizen should have the right to express any of their thoughts freely, and have the right and the duty to shoulder the repercussion of what they said as well. of As for the rest of us, we'll keep watching those whom use their "right" as a tool for exposing their internalized prejudice. Makes easier for us to distant ourselves from them if we want or better yet, use our own freedom of speech to correct the ignorant and to put the malicious to their place. 


\author{
ANEXO 3 \\ UNIVERSIDADE FEDERAL DE PERNAMBUCO \\ CENTRO DE ARTES E COMUNICAÇÃO \\ DEPARTAMENTO DE LETRAS \\ INGLÊS VI
}

In a democratic society, every citizen should have the right to express any of their thoughts freely

Around the world there are people who think that speaking their minds is right. We have come a long way, from dictatorial censorship, and fear of retaliation, not to use this acquired right as often as we please. Most countries in the world have suffered from some kind of dictatorship and now refuses to go back to this era of darkness.

Take Brazil for example, for about twenty years many people were tortured,
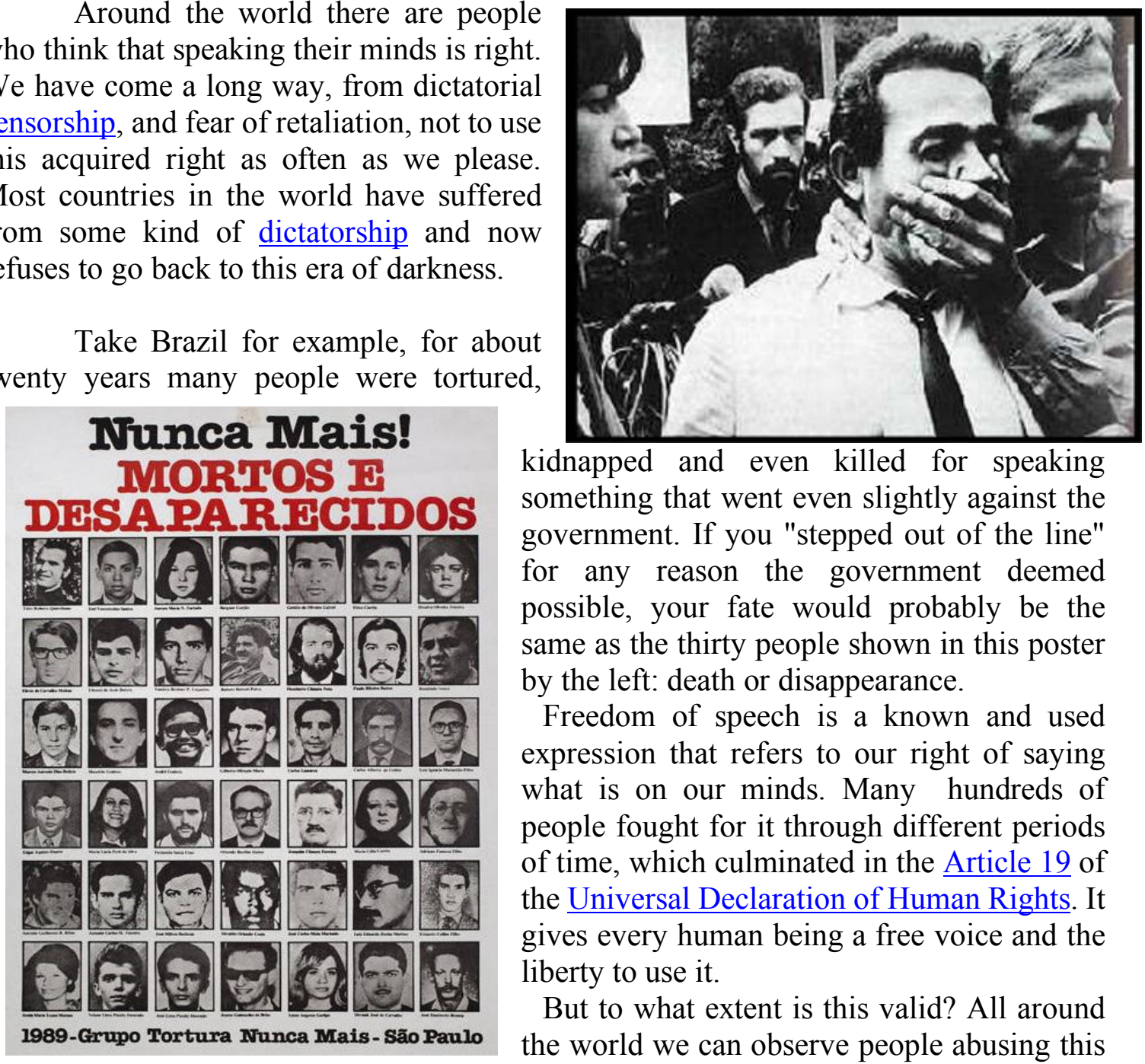

kidnapped and even killed for speaking something that went even slightly against the government. If you "stepped out of the line" for any reason the government deemed possible, your fate would probably be the same as the thirty people shown in this poster by the left: death or disappearance.

Freedom of speech is a known and used expression that refers to our right of saying what is on our minds. Many hundreds of people fought for it through different periods of time, which culminated in the Article 19 of the Universal Declaration of Human Rights. It gives every human being a free voice and the liberty to use it.

But to what extent is this valid? All around the world we can observe people abusing this right to hide Hate Speeches. Every citizen has the right to express any of their thoughts. However this does not mean they have a free pass concerning what is right or wrong. You are responsible for your words. And if your words hurt, offend, slander or vilifies someone, you also have the right to pay for what you have said.

One thing is someone having a different opinion from you, as for example, not liking certain kind of music. But from the moment a person says that that specific brand of music is made only by criminals, for instance, they are incurring in a crime of defamation. Hundreds of racist, homophobic, xenophobic, islamophobic and so forth, 
speeches are disguised as "Freedom of Speech" every day. A recent example is Europe's outbreak of hostility against Islam religion, inciting fear and hate against innocent muslins, all because of extremist attacks made by radicals. Even Hollywood helps perpetrate this stigma by making a blockbuster about a sniper (which according to his own biography enjoyed killing muslins) turned American hero.

So yes. Every citizen should have the right to express any of their thoughts freely, and have the right and the duty to shoulder the repercussion of what they said as well. As for the rest of us, we'll keep watching those whom use their "right" as a tool for exposing their internalized prejudice. Makes it easier for us to distant ourselves from them if we want or better yet, use our own freedom of speech to correct the ignorant and to put the malicious to their place. 